\title{
Penser l'éducation inclusive dans un contexte de discriminations et de diversité au Canada. L'importance de la pensée complexe d'Edgar Morin
}

Thinking Inclusive Education in a Canadian Context of Discriminations and Diversity. The Importance of Edgar Morin's Complex Thought

\section{Christian Bergeron}

\section{(2) OpenEdition}

Journals

\section{Édition électronique}

URL : http://journals.openedition.org/trema/6042

DOI : $10.4000 /$ trema. 6042

ISSN : 2107-0997

\section{Éditeur}

Faculté d'Éducation de l'université de Montpellier

\section{Référence électronique}

Christian Bergeron, «Penser l'éducation inclusive dans un contexte de discriminations et de diversité au Canada. L'importance de la pensée complexe d'Edgar Morin », Tréma [En ligne], 54 | 2020, mis en ligne le 01 décembre 2020, consulté le 14 décembre 2020. URL : http://journals.openedition.org/ trema/6042; DOI : https://doi.org/10.4000/trema.6042

Ce document a été généré automatiquement le 14 décembre 2020.

Trema 


\section{Penser l'éducation inclusive dans un contexte de discriminations et de diversité au Canada. L'importance de la pensée complexe d'Edgar Morin}

Thinking Inclusive Education in a Canadian Context of Discriminations and Diversity. The Importance of Edgar Morin's Complex Thought

\section{Christian Bergeron}

\section{Introduction}

1 Au moins depuis les années 1990 que les discriminations systémiques sont dénoncées en Ontario (Lewis, 1992). Malgré cela, les dénonciations n'ont pas mené à des transformations sociétales et institutionnelles importantes, car les discriminations systémiques sont encore présentes aujourd'hui, entre autres, dans les institutions publiques (Feireisen, 2020), le milieu universitaire (Bergeron, Blanchet \& LebonEyquem, 2020) et le milieu scolaire (Harris, 2020). Afin d'étudier cette problématique, nous nous intégrons comme observateurs dans notre observation (Morin, 2005). Nous sommes acteurs, sachant que nous sommes impliqués à titre de professeurs dans la formation à l'enseignement des futurs enseignants en langue française. Nous côtoyons les étudiants depuis un certain temps pour justifier l'importance d'être un observateur direct et parfois militant. Ainsi compris, le rôle du sociologue peut inclure une prise de parole "pour lutter contre toutes les formes de domination, y compris celles plus discrètes, plus dissimulées, mais non moins efficaces, qui caractérisent les rapports sociaux» (Paugam, 2008, p. 165). Enfin, sans pratiquer une sociologie de la critique (Boltanski, 1990), nous portons notre regard sociologique sur l'éducation inclusive, telle que pratiquée dans le système scolaire franco-ontarien (Ontario, Canada). Avant 
d'expliquer l'objectif de notre article, nous présentons un portrait sommaire du Canada et de l'Ontario au bénéfice du lecteur.

2 Le portrait culturel, linguistique, ethnique et spirituel est de plus en plus pluriel et contrasté au Canada (Bergeron, 2019). Aujourd'hui, deux enfants canadiens sur cinq sont issus de l'immigration (Statistique Canada, 2017b). De 2011 à 2016, un peu plus d'un million (1 212 075) de nouveaux immigrants se sont établis au Canada (Statistique Canada, 2017a). La province de l'Ontario, la plus peuplée et diversifiée du Canada, a accueilli en 2016 le plus d'immigrants (39\%), en comparaison avec le Québec (18\%) et la Colombie-Britannique (15\%). Les minorités visibles sont également très présentes en Ontario. Déjà en 2006, il y avait 2,7 millions d'Ontariens s'étant déclarés membres d'une minorité visible (Ministère de l'Éducation de l'Ontario, 2009). En 2016, ce sont environ 250 origines ethniques ou ascendances, dont 200 langues maternelles, qui ont été recensées au Canada. Conséquemment, quatre Canadiens sur dix ont mentionné détenir plus d'une ascendance (Statistique Canada, 2017b). Enfin, le pays d'origine des immigrants a passablement changé depuis quelques décennies. En 2016, 13,4\% des immigrants étaient nés en Afrique, alors qu'ils représentaient seulement 3,2\% de l'immigration en 1971 (Statistique Canada, 2017b). L'Afrique est maintenant le deuxième continent en importance, ayant surpassé l'Europe (3e place), mais loin derrière l'Asie (61,8\%). En somme, cette immigration plurielle et diversifiée influence de plus en plus la conception même du Canada.

3 Dans le cadre de cet article, nous présentons sommairement les résultats de $2018 \mathrm{du}$ test du Programme international pour le suivi des acquis des élèves (PISA) pour le Canada, ainsi que la problématique des discriminations en prévision de présenter les lignes directrices ministérielles de l'Ontario concernant les deux piliers de l'éducation inclusive, à savoir l'équité et la diversité. Avec cette stratégie politique, l'objectif « est de comprendre, d'identifier, d'examiner et d'éliminer les préjugés, les obstacles et la dynamique du pouvoir qui restreignent l'apprentissage et l'épanouissement des élèves et leur pleine contribution à la société » (Ministère de l'Éducation de l'Ontario, 2014, p. 6). Ensuite, de Les sept savoirs nécessaires à l'éducation du futur d'Edgar Morin (1999), nous étudions le chapitre IV : Enseigner l'identité terrienne ainsi que le chapitre VI : Enseigner la compréhension. Afin de bien comprendre la pensée de Morin, nous expliquons en parallèle la théorie de la complexité (Morin, 1994; 2005). La complexité, comme conception, prend en considération les contradictions, les antagonismes et les complémentarités qui façonnent notre compréhension de l'éducation du futur. En conclusion, nous interprétons la politique ontarienne sur l'éducation inclusive sous le prisme des savoirs nécessaires à l'éducation du futur. Penser l'éducation par le biais de la société n'a jamais été simple en raison de la complexification des rapports sociaux, des changements sociétaux continuels, des prises de conscience collective sur des problématiques sociales anciennes et nouvelles (écologie, violence, pauvreté, exclusion, discrimination, etc.), ainsi que de certains antagonismes (local vs globalité). Nous verrons ainsi comment l'éducation inclusive peut contribuer à l'éducation du futur.

4 «La diversité crée la complexité et la complexité crée la richesse » (Morin cité par Saez, 2008, p. 4). L'un des défis de la société canadienne est de tirer profit de cette diversité sur un continuum fondé "sur un dynamisme respectueux et une valorisation de toute la gamme de nos différences » (Ministère de l'Éducation de l'Ontario, 2014, p. 6). De fait, les origines ethniques et culturelles des Canadiens représentent un riche héritage pour la société, mais tout en complexifiant la définition du patrimoine culturel canadien, 
dont l'établissement d'une compréhension partagée du "vivre-ensemble » intégrant, entre autres, des valeurs fondamentales, telles que l'équité, la diversité et l'éducation inclusive. D'une manière précise, ce contexte complexe implique de revisiter les manières de penser l'éducation. L'éducation interpelle depuis un certain temps les décideurs politiques canadiens afin qu'ils développent un curriculum adapté à ces nouveaux paramètres sociétaux. Le ministère de l'Éducation de l'Ontario $(2009$; 2014 ; 2017) a émis au fil des récentes années des lignes directrices afin de promouvoir l'équité et l'éducation inclusive, car il existe dans les écoles des problématiques sociales de discrimination, de racisme et d'intimidation. Nous ajoutons à ces formes de discrimination, l'importance grandissante de la cyberviolence et du cyberharcèlement dont sont victimes les élèves (Hubert, 2014). L'une des solutions serait que « l'éducation inclusive veille à ce que tous les élèves se sentent représentés dans le curriculum et dans leur milieu immédiat, de même que dans leur milieu scolaire en général, et dans lequel la diversité est valorisée et toutes les personnes sont respectées » (Ministère de l'Éducation de l'Ontario, 2014, p. 97). À notre connaissance, il n'existe pas dans la littérature consacrée aux sciences de l'éducation, une analyse de l'éducation inclusive sous le prisme des savoirs nécessaires de l'éducation du futur selon Edgar Morin (1999). Ajoutons qu'il est difficile d'ignorer l'importance de la théorie de la complexité (Morin, 1994) pour saisir les subtilités de la complexité de la société canadienne plurilinguistique et pluriethnique, car «la compréhension d'autrui nécessite une conscience de la complexité humaine" (Morin, 1999, p. 55). Cette ouverture vers la pensée complexe de Morin nous permet d'interpréter l'éducation inclusive, sans pour autant éliminer certaines incertitudes inhérentes à l'étude d'un phénomène complexe et dynamique.

\section{Résultats au test PISA et discriminations}

5 Le programme PISA analyse le niveau scolaire, dans certaines matières (lecture, mathématique, science), des jeunes de 15 ans dans plus de 79 pays. Au dernier test PISA de 2018, de l'Organisation de coopération et de développement économiques (OCDE), le Canada s'est classé $6^{\mathrm{e}}$ (Radio-Canada, 2019). Les élèves canadiens performent très bien dans les mathématiques, les sciences ainsi que la lecture (compréhension de l'écrit). En matière d'équité, le Canada se situe dans la moyenne mondiale. Le nombre d'élèves canadiens issus de l'immigration est supérieur à la moyenne des pays de l'OCDE. En seulement 10 ans, le pourcentage d'élèves issus de l'immigration a augmenté significativement, de $24 \%$ en 2009 à $35 \%$ en 2019 (Radio-Canada, 2019). Le profil sociodémographique de la société canadienne évolue rapidement. Nous émettons l'hypothèse que cette augmentation de l'immigration peut expliquer, en partie, certaines tensions existantes dans les écoles. Par exemple, les résultats du test PISA montrent que l'intimidation à l'école est plus élevée au Canada (25\%) qu'ailleurs dans le monde $(23 \%)$. Près de trois élèves sur dix ont affirmé avoir été victimes de harcèlement au moins «quelques fois par mois ». Malgré les bons résultats du Canada au test PISA, le harcèlement ou l'intimidation chez les élèves a des conséquences néfastes: "le rejet, l'exclusion et l'isolement sont associés à des problèmes de comportement en classe, à un manque d'intérêt pour les études, à un faible niveau de rendement et à des taux de décrochage élevés » (Osterman, 2000 cité par ministère de l'Éducation de l'Ontario, 2009, p. 11). 
6 La problématique de l'intimidation est intimement reliée à celle de la discrimination. Cette problématique n'est pas nouvelle au Canada. Déjà en 2005, la Cour suprême du Canada reconnaissait « que le préjugé racial contre les minorités visibles est si notoire et incontestable que son existence sera admise sans preuve à l'appui » et doit être traitée comme un «fait social» (cité par ministère de l'Éducation de l'Ontario, 2009, p. 9). Malgré tous les efforts déployés par le gouvernement, les préjugés discriminatoires et les obstacles systémiques demeurent problématiques. En 2007, la Commission ontarienne des droits de la personne a spécifié que le nombre de plaintes liées à la couleur de la peau et à un handicap dépassait de loin le nombre de plaintes liées à d'autres formes de discriminations (Ministère de l'Éducation de l'Ontario, 2014). Un récent rapport, toujours publié par la Commission ontarienne des droits de la personne (2017, p. 2), «questionnait les gens à propos de leurs connaissances des droits de la personne, de leurs attitudes envers divers groupes et de leurs expériences personnelles en matière de discrimination. " Les résultats montrent que sept personnes sur dix pensent que le racisme est une problématique importante en Ontario. Sur les 1500 adultes sondés, les principaux motifs de discrimination mentionnés sont la couleur de la peau (63\%), l'orientation sexuelle ( $34 \%$ ), le handicap ( $25 \%)$ et la croyance/religion (24\%). Les discriminations ont surtout lieu au travail ( $45 \%)$, dans les lieux publics (42\%) et à l'école (12\%). Concernant les représentations sociales des participants, des stéréotypes négatifs existent à l'endroit des récipiendaires de l'aide sociale (61\%), des personnes d'origine arabe (56\%), des musulmans $(55 \%)$ des personnes transgenres $(54 \%)$ et des réfugiés $(54 \%)$. Terminons sur une piste de solutions possibles aux discriminations : neuf participants sur dix s'accordent à dire que les élèves devraient en apprendre davantage à l'école sur les droits de la personne (Commission ontarienne des droits de la personne, 2017). Dans ce contexte sociétal, le fait d'accentuer les efforts dans le développement de l'équité et de l'éducation inclusive semble primordial.

\section{La stratégie de l'Ontario en matière d'équité et d'éducation inclusive}

7 Le ministère de l'Éducation de l'Ontario (2009) a développé une politique afin de donner « une » chance à tous les élèves d'atteindre tout leur potentiel. Pour atteindre cet objectif, les 72 conseils scolaires ontariens (catholique romain francophone/catholique romain anglophone ainsi que public francophone/public anglophone) doivent adhérer à une même vision, à savoir que tous les élèves, les parents ainsi que les acteurs des milieux scolaires se sentent acceptés et respectés pour ce qu'ils sont, dans leur diversité (ethnique, culturelle, linguistique, sexuelle, handicap, etc.). L'un des objectifs est d'éveiller les élèves à l'altérité et de créer une société plus équitable et soucieuse du bien-être de chacun. Afin de pérenniser ce plan d'action, le gouvernement a créé le Secrétariat de l'équité en matière d'éducation. Ce secrétariat a le mandat d'apporter « de nouvelles ressources pour cerner et éliminer les obstacles systémiques afin de protéger les intérêts des élèves, des familles et du personnel des écoles de l'Ontario, et de favoriser leur autonomisation» (Ministère de l'Éducation de l'Ontario, 2017, p. 10). Comme nous le verrons, l'éducation inclusive est un processus politique et organisationnel qui s'inscrit sur la longue durée avec la collaboration de tout un chacun impliqué de près ou de loin dans le système d'éducation. 
Cette politique s'organise autour de trois piliers: diversité - équité - éducation inclusive. La diversité des qualités humaines et les attributs d'un groupe ou d'une communauté ont trait à «l'ascendance, à la culture, à l'origine ethnique, au sexe, à l'identité sexuelle, à l'identité fondée sur le genre, à l'orientation sexuelle, à la langue, aux capacités physiques ou intellectuelles, à la race, à la religion et au statut socioéconomique ». L'équité réfère au "principe de traitement juste, inclusif et respectueux de toutes les personnes " (Ministère de l'Éducation de l'Ontario, 2009, p. 6). L'éducation inclusive, quant à elle, est basée sur les principes d'acceptation et d'inclusion de tous les élèves. L'éducation inclusive englobe et encadre la diversité et l'équité. Elle s'assure que tous les élèves trouvent leur place dans le milieu scolaire et se sentent représentés dans le curriculum. Ainsi, toutes les personnes sont respectées dans leur spécificité, sans négliger pour autant le tout. En somme, ces trois piliers accentuent le trait sur l'importance de (re)valoriser le sens de la justice, de l'équité et du respect dans les écoles. Pour schématiser, l'éducation inclusive :

- Est à la base de l'excellence :

9 Dans une société diversifiée, l'équité est un élément fondamental contribuant à l'excellence en éducation et à de hauts niveaux de rendement des élèves.

- Répond aux besoins individuels :

10 L'équité ne signifie pas un traitement identique pour tous les élèves. Bien au contraire, il s'agit de combler les besoins individuels de chaque élève et de fournir les conditions nécessaires et les mesures d'intervention requises pour l'aider à réussir.

- Reconnaît et élimine les obstacles :

11 Tous les élèves sont appuyés équitablement grâce à l'identification et à l'élimination des barrières discriminatoires qui les empêchent de réaliser leur plein potentiel.

- Encourage un sentiment d'appartenance :

Offrir une éducation équitable et inclusive est primordial pour procurer à chaque enfant un sentiment de bien-être.

- Met à contribution la communauté dans son ensemble :

13 Des partenariats efficaces et significatifs entre écoles et communautés constituent une composante essentielle d'un système d'éducation reposant sur l'équité et l'inclusion.

-Vient prolonger et améliorer les initiatives existantes ou antérieures :

14 Des recherches claires et des analyses comparatives menées sur des politiques et pratiques réussies sont à la base du développement et du partage de ressources.

- Se manifeste dans l'ensemble du système :

Le ministère de l'Éducation, les conseils scolaires et les écoles intégreront des principes d'équité et d'éducation inclusive dans tous leurs programmes, politiques et pratiques (Ministère de l'Éducation de l'Ontario, 2014, p. 16).

16 Le principe fédérateur de l'éducation inclusive est que les écoles doivent être des lieux d'apprentissage où les élèves font l'expérience qu'ils sont représentés, entre autres, dans le curriculum et que cette diversité se reflète également au sein des enseignants et du personnel. L'équité et la diversité deviennent la base de la réussite scolaire tout en encourageant le sentiment d'appartenance à l'école. Cependant, cinq ans après la mise en place de cette politique, le constat est le suivant : il faut constamment y mettre des efforts, car les préoccupations sont complexes et changeantes dans les communautés et 
les écoles (Ministère de l'Éducation de l'Ontario, 2014). Par exemple, des discriminations systémiques co-existent au quotidien dans les écoles. Les discriminations systémiques sont issues normalement «de politiques ou pratiques institutionnelles apparemment neutres, mais renforcées par certaines structures institutionnelles et dynamiques de pouvoir, et qui se traduisent en pratique par le traitement différentiel et non équitable des membres de certains groupes » (Ministère de l'Éducation de l'Ontario, 2014, p. 95). Malgré la volonté politique d'éliminer les discriminations, ces dernières témoignent que le politique, le système scolaire, les intervenants et les communautés doivent prendre conscience, ensemble, des structures existantes de pouvoir qui perpétuent les formes d'iniquités et de discriminations. Les obstacles peuvent être nombreux en raison de la multiplicité des possibles formes de discriminations fondées sur le sexe, l'orientation sexuelle, l'identité sexuelle, la race, l'ethnicité, la religion/spiritualité, le statut socioéconomique, les capacités physiques ou intellectuelles, etc. (Ministère de l'Éducation de l'Ontario, 2014). Avec l'éclatement des mœurs, des normes, des modes de vie ainsi que de l'accroissement significatif d'une immigration diversifiée (autrefois, majoritairement européenne et chrétienne), les formes de discriminations évoluent et se complexifient également. En d'autres mots, la diversité de la société canadienne, perçue comme une richesse, est également l'objet de rejet, de violence et de discriminations systémiques. Afin d'éliminer les discriminations et de rendre les écoles davantage équitables, l'éducation du futur de Morin (1999) nous semble nécessaire, tout en améliorant notre compréhension de la théorie de la complexité appliquée à l'éducation inclusive. Effectivement, « si vous avez le sens de la complexité vous avez le sens de la solidarité. De plus, vous avez le sens du caractère multidimensionnel de toute réalité » (Morin, 2005, p. 92).

\section{Les savoirs nécessaires à l'éducation du futur d'Edgar Morin}

17 À l'aube du XXIe siècle, l'UNESCO a interpellé Edgar Morin afin qu'il rédige un rapport sur l'éducation du futur. De ses analyses découlent sept savoirs nécessaires pour l'éducation du futur, à savoir 1) les cécités de la connaissance, 2) les principes d'une connaissance pertinente, 3) l'enseignement de la condition humaine, 4) l'enseignement de l'identité terrienne, 5) l'affrontement des incertitudes, 6) l'enseignement de la compréhension et 7) l'éthique du genre humain. Nous expliquons en détail les deux principaux savoirs nécessaires en lien avec l'éducation inclusive, c'est-à-dire l'enseignement de l'identité terrienne et l'enseignement de la compréhension. Nous commençons par détailler la théorie de la complexité pour ensuite l'intégrer dans la présentation de ces deux savoirs.

\section{Théorie de la complexité}

La pensée complexe de Morin nous interpelle à ne pas avoir recours à la simplification du monde en raison de sa trop grande complexité. Selon Morin (1994, p. 323), «la pensée complexe doit dépasser les entités closes, les objets isolés, les idées distinctes, mais aussi ne pas se laisser enfermer dans la confusion, le flou, l'ambiguïté, la contradiction.» La notion «complexe» est polysémique. Dans la théorie de la complexité, cette notion « relève d'une part du monde empirique, de l'incertitude, de 
l'incapacité d'être certain de tout, de formuler une loi, de concevoir un ordre absolu. Il relève d'autre part de quelque chose de logique, c'est-à-dire de l'incapacité d'éviter des contradictions» (Morin, 2005, p. 92). Il développe trois principes qui aident à penser la complexité, à savoir le principe dialogique, la récursion organisationnelle et le principe hologrammatique :

1. Le principe dialogique. Ce processus nous permet de maintenir la dualité au sein de l'unité. Il associe deux termes à la fois complémentaires et antagonistes [ordre et désordre] ;

2. La récursion organisationnelle. Un processus récursif est un processus où les produits et les effets sont en même temps causes et producteurs de ce qui les produit. On retrouve l'exemple de l'individu, de l'espèce et de la reproduction. Nous, individus, nous sommes les produits d'un processus de reproduction qui est antérieur à nous. Mais une fois que nous sommes produits, nous devenons les producteurs du processus qui va continuer. La société est produite par les interactions entre individus, mais la société produite, rétroagit sur les individus et les produits ;

3. Le principe hologrammatique. L'idée donc de l'hologramme dépasse, et le réductionnisme qui ne voit que les parties et le holisme qui ne voit que le tout. Alors on peut enrichir la connaissance des parties par le tout et du tout par les parties, dans un même mouvement producteur de connaissances. Donc l'idée hologrammatique est elle-même liée à l'idée récursive, qui elle-même est liée à l'idée dialogique en partie (Morin, 2005, p. 99-101).

19 En somme, la pensée complexe est dialogique (ordre/désordre/organisation), récursive et hologrammatique, mais «le cœur de la complexité, c'est l'impossibilité et d'homogénéiser et de réduire, c'est la question de l'unitas multiplex» (Morin, 2005, p. 141). De fait, la pensée complexe est le noyau de la reproduction (principe récursif) entre le simple et le complexe (principe hologrammatique) parce que cette relation est autant contradictoire, antagoniste et complémentaire (principe dialogique).

\section{Enseigner l'identité terrienne}

Le trésor de l'humanité est dans sa diversité créatrice, mais la source de sa créativité est dans son unité génératrice (Morin, 1999, p. 35).

L'enseignement de l'identité terrienne est de comprendre a priori le monde dans sa complexité, dans le sens qu'il faut étudier à la fois l'unité et la diversité du monde, ses contradictions, ses antagonismes et ses complémentarités. Ainsi, l'humanité trouve sa source de créativité dans une forme d'unité génératrice. Pour Morin, il n'y a pas de centre organisateur, de tour de contrôle, de l'organisation du monde dans sa verticalité via un système global. Le monde est en interdépendance, en interrelation avec ses parties: « chaque partie du monde fait de plus en plus partie du monde, et le monde, en tant que tout, est de plus en plus présent en chacune de ses parties. Cela se vérifie non seulement pour les nations et les peuples mais aussi pour les individus » (Morin, 1999, p. 35). Les liens d'interdépendances entre l'individu et la mondialisation témoignent à la fois de forces antagonistes (mouvements de résistance contre la mondialisation, retour de thèses nationalistes, conflits entre nations, entre modernité et tradition, entre les riches et les pauvres, entre laïcité et religion, etc.) et de complémentarité (marché mondial de consommation, moyen de transport et de communication globalisés, déterritorialisation des relations, etc.).

21 Avec la mondialisation, le monde se rétrécit et les nations sont de plus en plus interdépendantes (Nations Unies, 2013). D'une part, l'identité terrienne est en lien avec le principe hologrammatique de la pensée complexe, car « chaque individu reçoit en lui 
ou consomme les informations et les substances venant de tout l'univers" (Morin, 1999, p. 35). En quelque sorte, le moi individuel est à la fois multiple, divisé et hétérogène, mais tout en gardant une unité (le un et le multiple de la pensée complexe, Morin \& Le Moigne, 1999). D'autre part, toujours selon la pensée complexe, Morin fait référence à une relation auto-écologique du développement identitaire humain. En lien avec le principe dialogique, nos relations peuvent être «à la fois d'opposition/ distinction et d'implication/intégration, d'altérité et d'unité. [...] Le propre d'un être vivant, c'est de se distinguer, par son individualité et sa singularité, des autres êtres et de son environnement " (Morin, 1994, p. 328). Les antagonismes et les formes de complémentarités sont essentiels dans la construction identitaire terrienne.

\section{Enseigner la compréhension}

Enseigner la compréhension entre les personnes comme condition et garant de la

solidarité intellectuelle et morale de l'humanité (Morin, 1999, p. 51).

«La communication n'apporte pas la compréhension » (Morin, 1999, p. 52). Déjà, en 1999, Morin avait présagé les incompréhensions que la communication créerait. C'est un lieu commun d'affirmer que les technologies d'informations et de communications (TIC), incluant les réseaux sociaux, ont favorisé grandement le développement de nouveaux modes de communication planétaire. Il semble que la démultiplication des moyens de communiquer (trop-plein de communication ; $c f$. Robert, 2009) n'a toutefois pas été accompagnée par une augmentation de la compréhension. Au contraire, il semble exister aujourd'hui une plus grande incompréhension (infox ("fake news»), théories conspirationnistes ou complotistes ; $c f$. Sauvageau, Thibault et Trudel, 2018) et une cyberviolence dans les propos (intimidation, harcèlement, discrimination; $c f$. Blaya, 2011). À travers, entre autres, les TIC et les réseaux sociaux s'expriment l'égocentrisme, l'ethnocentrisme et le sociocentrisme. L'égocentrisme consiste à se tromper par « l'autojustification, l'autoglorification et la tendance à rejeter sur autrui, étranger ou non, la cause de tous maux » (Morin, 1999, p. 53). Comme nous l'avons montré, certaines catégories d'individus ou de communautés sont victimes de stéréotypes négatifs. Cet égocentrisme témoigne d'une incompréhension de soi qui est à la fois « une source très importante de l'incompréhension d'autrui. On se masque à soi-même ses carences et faiblesses, ce qui rend impitoyable pour les carences et faiblesses d'autrui »(Morin, 1999, p. 53). Pour sortir de cet égocentrisme, il faut procéder à un auto-examen critique afin de provoquer une décentration individuelle et ainsi reconnaître ses limites, ses faiblesses et cesser d'être juge de toutes choses (Morin, 1999). L'ethnocentrisme et le sociocentrisme, quant à eux, "nourrissent les xénophobes et racismes et peuvent aller jusqu'à retirer à l'étranger la qualité d'humain » (Morin, 1999, p. 53). L'existence des discriminations systémiques en est malheureusement l'expression de ces manques de compréhension et d'humanité. Par exemple,

les idées préconçues, les rationalisations à partir de prémisses arbitraires, l'autojustification frénétique, l'incapacité de s'autocritiquer, le raisonnement paranoïaque, l'arrogance, le déni, le mépris, la fabrication et la condamnation de coupables sont les causes et les conséquences des pires incompréhensions issues à la fois de l'égocentrisme et de l'ethnocentrisme (Morin, 1999, p. 53).

Dans ce savoir nécessaire du nouveau millénaire, nous retrouvons l'expression des principes (dialogique, récursif et hologrammatique) de la pensée complexe (Morin, 1994). Les modes de communication, antagonistes et complémentaires, sont ainsi le produit des interactions entre les personnes (égocentrisme, ethnocentrisme, 
sociocentrisme), mais ces modes de communication rétroagissent sur les personnes et les produisent à leur tour. En d'autres mots, plus un mode de communication devient le fruit des interactions égo-ethno-socio-centristes des personnes, plus ce dernier rétroagira sur les personnes et les définira. Ce qui explique que certaines personnes abandonnent, entre autres, les réseaux sociaux (Colombain, 2019) afin de favoriser une compréhension humaine intersubjective des relations terriennes.

Si une communication est bien transmise et comprise, elle apporte l'intelligibilité, mais elle est insuffisante pour la compréhension. Selon Morin (1999), l'enseignement de la compréhension se décline sous deux dimensions : la compréhension intellectuelle (ou objective) et la compréhension humaine intersubjective. D'une part, «comprendre signifie intellectuellement appréhender ensemble, com-prehendere, saisir ensemble (le texte et son contexte, les parties et le tout, le multiple et l'un). La compréhension intellectuelle passe par l'intelligibilité et par l'explication » (Morin, 1999, p. 51). D'autre part, la compréhension humaine intersubjective est «un processus d'empathie, d'identification et de projection. Toujours intersubjective, la compréhension nécessite ouverture, sympathie, générosité» (Morin, 1999, p. 52). L'enseignement de la compréhension exige de comprendre les causes de l'incompréhension de la communication, dans un élan d'ouverture, en passant d'une métastructure de pensée à une autre afin de la dépasser.

\section{Interprétation de l'éducation inclusive}

L'éducation inclusive est basée sur les principes d'acceptation et d'inclusion de tous les élèves. Elle englobe et encadre à la fois la diversité et l'équité (Ministère de l'Éducation de l'Ontario, 2014). Elle s'assure ainsi que tous les élèves trouvent leur place à l'école et se sentent représentés dans le curriculum. L'équité et la diversité deviennent donc la base de la réussite scolaire tout en encourageant le sentiment d'appartenance à l'école. Cependant, la diversité des personnes dans la société canadienne, perçue comme une richesse, est également l'objet de rejet, de violence et de discriminations systémiques. Ces obstacles sont nombreux en raison de la multiplication des minorisations individuelles et collectives et ces obstacles peuvent prendre la forme de discriminations fondées sur le sexe, l'orientation sexuelle, l'identité sexuelle, la couleur de la peau, l'ethnicité, la religion/spiritualité, le statut socioéconomique, les capacités physiques ou intellectuelles, la langue, etc. (Ministère de l'Éducation de l'Ontario, 2014). Pour nous aider dans notre analyse de l'éducation inclusive, nous avons recours à la pensée complexe ainsi qu'aux deux savoirs nécessaires de Morin pour l'éducation du futur.

Dans nos tâches d'enseignement, notre pédagogie est active et participative (horizontalité des échanges) et elle s'appuie sur l'inclusion de la diversité, autant dans la matière enseignée que dans les discussions de groupes, et ce, dans le respect des communautés franco-ontariennes (francophones de l'Ontario) ${ }^{1}$. Par exemple, nous enseignons l'histoire du système scolaire franco-ontarien et nous sensibilisons les étudiants aux difficultés de l'enseignement en langue française dans une province anglophone. Nous traitons de l'importance de la construction identitaire francophone, tout en y intégrant les diverses formes de la Francophonie. Nous réfléchissons aussi avec les étudiants sur les défis et enjeux des populations immigrantes. Par exemple, le manque de diversité dans les institutions (Ratel, 2020) ainsi que les difficultés d'intégration des communautés noires (Radio-Canada, 2020) sont des réalités qui sont 
partagées par les étudiants. D'ailleurs, nous outillons les étudiants sur l'intersectionnalité afin qu'ils soient en mesure de mieux comprendre et de lutter contre les discriminations qu'ils peuvent subir ou voir dans les écoles. Enfin, nous débattons en classe des modèles d'intégration des populations immigrantes (multiculturalisme canadien vs interculturalisme québécois ${ }^{2}$ ), car nous sommes confrontés quotidiennement aux contradictions, antagonismes et complémentarités de ces derniers. En raison de la proximité géographique (moins de deux kilomètres séparent les centres-villes d'Ottawa et de Gatineau), les étudiants vivent au Québec et en Ontario. Entre ces deux provinces, elles n'ont pas toujours la même conception de l'inclusion des immigrants, elles n'ont parfois pas la même compréhension de la laïcité et des rapports avec les religions et enfin, l'une est majoritairement francophone (culture d'origine française) et l'autre anglophone (culture anglo-saxonne). La perception de l'existence des deux solitudes entre le Québec et les autres provinces nous semble toujours d'actualité ( $c f$. MacLennan, 1945). En d'autres mots, les difficultés de communication entre le Québec et le Canada sont toujours présentes et elles surviennent dans les salles de classe. De part et d'autre, ils doivent "s'ouvrir à cette diversité et dépasser les étapes de la tolérance et de la satisfaction pour aller vers l'inclusion et le respect " (Ministère de l'Éducation de l'Ontario, 2014, p. 6), tout en acceptant que soient exprimés des idées, des convictions et des choix contraires aux nôtres (Morin, 1999).

Les opposants, à l'un ou l'autre des deux modèles (multiculturalisme et interculturalisme), ne cherchent pas à dépasser les contradictions et les antagonismes de ces derniers pour l'enseignement de la compréhension (cf. Bock-Côté, 2016). Peu importe le modèle préférentiel du "vivre-ensemble», il est préférable, comme le précise Maalouf $(2019$, p. 83), à nous « efforcer de prendre en compte les nombreuses sensibilités qui existent au sein de la population, de sorte que chaque citoyen se reconnaisse dans la société où il vit, dans son système social et ses institutions. » Ce qui implique de respecter ceux et celles qui préfèrent vivre dans l'un ou l'autre des modèles d'inclusion de la diversité, et ce, sans rejeter l'Autre. Ce que Maalouf (1998) nommera le principe de réciprocité. La réciprocité est importante dans l'éducation à l'altérité. Les futurs enseignants sont appelés à réfléchir au fait qu'ils peuvent être à la fois porteurs d'inclusion et d'exclusion (cf. égocentrisme, ethnocentrisme; Morin, 1994). Selon la pensée complexe, "nous pouvons commencer à considérer l'unité si troublante de l'identité individuelle, qui s'affirme à la fois, d'une part par égo-référence et égocentrisme, d'autre part par référence à un héritage antérieur venu d'ailleurs, usé et utilisé par d'autres vies, produit et reproduit par d'autres générations » (Morin, 1994, p. 293). En Ontario, l'immigration est importante et diversifiée, sans oublier l'existence des communautés franco-ontariennes qui ont, quant à elles, des héritages particuliers ancrés dans un contexte linguistique minoritaire. En conséquence, les références à des héritages antérieurs sont plurielles et contrastées. D'une part, ces référents peuvent conduire à l'exclusion, voire au rejet des différences. D'autre part, au contraire, ces mêmes référents peuvent être une richesse pour la Francophonie et le Canada.

\section{Enseigner l'identité terrienne dans l'éducation inclusive}

Reprenons l'importance de l'enseignement de l'identité terrienne de Morin (1999) afin d'analyser l'éducation inclusive. L'éducation inclusive accorde une grande importance 
à la construction identitaire, contextualisée à la réalité des communautés francophones qui vivent en contexte linguistique minoritaire. La construction identitaire est fortement enracinée dans la préservation de la culture franco-ontarienne, tout en y incluant les autres francophones ou francophiles venus d'ailleurs du Canada ou du monde. Il est à propos de favoriser la culture d'origine d'un lieu précis pour plusieurs raisons. "Tout être humain, toute collectivité doit irriguer sa vie par une circulation incessante entre son passé où il ressource son identité en se rattachant à ses ascendants, son présent où il affirme ses besoins et un futur où il projette ses aspirations et ses efforts » (Morin, 1999, p. 41). Cette circulation, ce principe récursif, est nécessaire dans le déploiement d'une pensée complexe, tout en l'ouvrant au dialogue, car plusieurs personnes se rattachent à différents ascendants, mais tout en partageant et produisant la société canadienne. Afin de faciliter l'enseignement de l'identité terrienne, une nécessaire décentralisation des cultures, des origines, des valeurs sont nécessaires, tout en reconnaissant des carences, des limites dans notre capacité à comprendre et à interagir avec l'Autre.

L'enseignement de l'identité terrienne passe également par une compréhension des relations entre la personne (son individualité) et la mondialisation. Il est difficile en Ontario de concevoir la construction identitaire sans l'accompagner d'une réflexion du processus planétaire, des mouvements de population et d'immigration. Cela n'empêche pas qu'il existe des conflits, des résistances, du racisme, de la xénophobie, de l'islamophobie, etc. Le multiculturalisme canadien, dans sa tentative d'unification des différentes communautés culturelles, s'accompagne de son contraire, c'est-à-dire la balkanisation: «le monde devient de plus en plus un, mais il devient en même temps de plus en plus divisé » (Morin, 1999, p. 36). Toujours selon Morin, l'éducation doit agir comme une passerelle (hologrammatique) entre l'ancien et l'ouverture d'esprit nécessaire pour intégrer le nouveau. La rencontre entre l'ancien et le nouveau doit déboucher vers un renouvellement de l'éducation, sans annihiler les nouvelles forces issues des élèves et ni en les abandonnant à eux-mêmes. De fait, l'éducation inclusive doit « combler les besoins individuels de chaque élève et de fournir les conditions nécessaires et les mesures d'intervention requises pour l'aider à réussir » (Ministère de l'Éducation de l'Ontario, 2014, p. 16). L'un des enjeux est de déterminer comment répondre aux besoins fortement différenciés des élèves, sans y établir une quelconque forme de hiérarchisation entre eux.

\section{Enseigner la compréhension dans l'éducation inclusive}

À l'ère des communications virtuelles et jugées parfois déshumanisantes (Colombain, 2019). La valorisation de l'enseignement de la compréhension devient une priorité. Il n'est pas étonnant de noter l'importance contemporaine de l'éducation inclusive dans cette conscientisation de la rencontre avec l'Autre. L'enseignement de la compréhension est donc plus que nécessaire (Morin, 1999). Un fait surprenant de cette politique ministérielle (Ministère de l'Éducation de l'Ontario, 2014), c'est que l'enseignement de la compréhension n'est pas valorisé explicitement. Pourtant, sans compréhension, il est impossible d'utiliser une pédagogie inclusive. De plus, le mode d'intégration des populations immigrantes n'est pas abordé dans la politique. Il est tenu pour acquis qu'il existe un seul modèle possible en Ontario, c'est-à-dire le 
multiculturalisme. Ce qui représente une grande limite à l'expression (inclusion) des diverses formes de compréhension de la complexité de la diversité.

$\mathrm{Au}$ Canada (excluant le Québec) se déclinent trois grandes valeurs, à savoir le multiculturalisme, les droits de la personne et la diversité. Ces valeurs peuvent se comprendre au prisme de l'enseignement de la compréhension. La diversité est intimement associée au multiculturalisme canadien, à laquelle les différences sont légalement respectées et culturellement valorisées, et ce, dans le souci du respect des droits et libertés des personnes. Ces trois valeurs sont en quelque sorte le socle de la société canadienne. Cependant, il semble exister une contradiction entre les valeurs, $a$ priori, déjà enracinées dans la société canadienne et le besoin de développer une stratégie sur l'inclusion et la diversité. L'un des pièges est d'associer le multiculturalisme à la diversité. De fait, « une reconnaissance du pluralisme qui se limite à la diversité de groupe apparaît comme une conception essentialiste de l'identité et néglige la diversité résultant de l'expression de la pluralité des individus » (Gignac, 1997, p. 36). En d'autres mots, le multiculturalisme est composé de la diversité des identités plurielles, mais il n'implique pas une réelle inclusion des différences et ni une reconnaissance des inégalités à l'intérieur des diverses communautés (Hikido \& Murray, 2015 cités dans Bélanger, 2019). En conséquence, les discriminations sont importantes, ce qui renforce le besoin de l'enseignement de la compréhension, dont l'inclusion du principe dialogique de la pensée complexe. Bref, le multiculturalisme n'est pas la forme aboutie de l'inclusion de la diversité dans la société canadienne. Si c'était le cas, il n'y aurait pas autant de discriminations systémiques depuis plusieurs années (cf. Lewis, 1992).

Concernant les obstacles à la compréhension, Morin (1999, p. 53) explique que ces derniers «sont non seulement l'indifférence mais aussi l'égocentrisme, l'ethnocentrisme, le sociocentrisme qui ont pour trait commun de se mettre au centre du monde et de considérer soit comme secondaire, insignifiant ou hostile tout ce qui est étranger ou éloigné ». Conséquemment, certaines catégories de personnes sont plus discriminées que d'autres en raison qu'elles peuvent être jugées comme «secondaire, insignifiante ou hostile » à la société. À ce titre, il est difficile de faire soustraction des discriminations que vivent également les communautés autochtones des Premières Nations, Métis et Inuits (Lepage, 2019; Yvon, 2020) ainsi que les communautés francophones hors-Québec (LeVasseur, 2016 ; Vachet, 2020). Selon Maalouf (2019, p. 83),

ni dans le déni des différences, qui dissimule les problèmes et contribue souvent à les aggraver ; mais dans l'instauration d'un dispositif de vigilance, au travers duquel on prendrait soin de vérifier en permanence qu'aucun secteur de la population, et même, idéalement, aucun citoyen, ne soit victime d'une discrimination injuste liée à la couleur, à la religion, à l'ethnie, à l'âge, au sexe.

Un dispositif de vigilance est important dans l'éducation inclusive, car les discriminations systémiques sont bien enracinées dans les institutions et la société canadienne (Ministère de l'Éducation de l'Ontario, 2014). Plusieurs discriminations peuvent se recouper et générer des obstacles pour certaines personnes jugées selon leurs caractéristiques individuelles (Bergeron, Blanchet \& Lebon-Eyquem, 2020). Sans développer longuement ce paradigme analytique, l'intersectionnalité est pertinente dans l'étude des minorisations/discriminations (Garneau, 2010). Précisons tout de même que l'intersectionnalité recoupe au moins deux ou plusieurs formes de discriminations, dont une personne «peut faire simultanément l'objet, créant ainsi des obstacles ou des partis pris supplémentaires empêchant cette personne ou ce groupe 
d'être traité avec équité " (Ministère de l'Éducation de l'Ontario, 2014, p. 98). Ces différentes formes de discriminations ou encore, de la présence du harcèlement ou de l'intimidation dans les écoles témoignent d'une conception homogène de la société ; la pluralité/diversité des individus est niée malgré le fait que le Canada est un pays multiculturel. Sachant que le multiculturalisme s'approprie les vertus de la diversité, il est parfois difficile d'admettre que ce modèle cache en fait un système de discriminations systémiques (Davis citée dans Slaughter \& Singh, 2020). Maalouf (2019, p. 52) parlera alors du «mythe pervers de l'homogénéité ». Ce mythe, Morin (1999, p. 54) le retrouvera dans « le mode de penser dominant, réducteur et simplificateur, allié aux mécanismes d'incompréhension, qui détermine la réduction d'une personnalité, multiples par nature, à l'un seul de ses traits. » Nous l'observons, la pensée complexe de Morin nous aide à mieux analyser les obstacles à l'éducation réellement inclusive et d'être critique envers le multiculturalisme.

\section{Conclusion}

La société canadienne évolue rapidement et il devient de plus en plus complexe d'évaluer les besoins des populations immigrantes ainsi que ceux des populations canadiennes. La théorie de la complexité (Morin, 2005 ; 1994 ; Morin \& Le Moigne, 1999) nous aide à interpréter l'éducation inclusive, tout en l'enracinant dans les savoirs nécessaires du nouveau millénaire. Une incertitude persiste tout de même: cette pédagogie sera-t-elle en mesure d'éradiquer à long terme les diverses formes de discriminations? La pensée complexe nous aide dans la capabilité d'accepter l'incertitude quant à la solution de l'éducation inclusive. La pensée complexe, «c'est la pensée apte à relier, contextualiser, globaliser, mais en même temps à reconnaître le singulier, l'individuel, le concret» (Morin \& Le Moigne, 1999, p. 266). La conscientisation de l'importance de l'enseignement à l'identité terrienne et de l'enseignement de la compréhension est un moyen concret de relier l'un et le multiple (unitas multiplex), l'individu et l'Autre, le national et la mondialisation, et enfin, de contextualiser le contexte canadien vis-à-vis ses défis quant à l'intégration des populations immigrantes, et ce, au-delà des "promesses » du multiculturalisme. Des défis de compréhension qui concernent aussi le monde. "La seule vraie mondialisation qui serait au service du genre humain est celle de la compréhension, de la solidarité intellectuelle et morale de l'humanité » (Morin, 1999, p. 57).

35 En nous inspirant de La ferme des animaux de Georges Orwell (1984), nous pouvons résumer ainsi l'un des enjeux de l'éducation inclusive : «tous les élèves sont égaux, mais certains le sont plus que d'autres $»^{3}$. Des catégories de personnes, en raison de leurs différences, sont davantage discriminées dans la société canadienne. De même, une forme de hiérarchisation des rapports sociaux existe entre les groupes minoritaires (Garneau, 2010). Enfin, « cette représentation de la diversité, uniquement associée à des élèves qui viennent d'ailleurs, risque de contribuer, par la stigmatisation qui peut en découler, à créer ce qu'il s'agit d'éviter » (Bélanger, 2019, p. 124). La compréhension des discriminations systémiques prend tout son sens dans l'interprétation de la persistance de cette problématique, et ce, malgré tous les efforts effectués depuis de nombreuses années par le ministère de l'Éducation de l'Ontario, des conseils scolaires, des enseignants et des communautés. Cela dit, la pensée complexe nous enseigne qu'il ne faut pas s'enfermer dans une forme de déterminisme contemporain, « c'est-à-dire dans 
la croyance que ce qui se passe maintenant va continuer indéfiniment » (Morin, 2005, p. 110). Sans être une recette miracle, l'éducation inclusive peut jouer un rôle actif dans l'élimination des discriminations.

La diversité est une richesse pour le monde (UNESCO, 2001). En conséquence, essayer d'exclure certaines catégories d'individus déshumanise notre société (Maalouf, 2019). Notre société doit, au contraire, comprendre l'importance pour elle-même d'intégrer la diversité, des éléments extérieurs, pour entretenir sa propre vitalité (Saez, 2008). La recherche d'homogénéisation, au nom de l'unité nationale ou encore, de la protection des populations locales n'a pas "pour conséquence de conforter l'égalité entre les citoyens ou d'abolir les discriminations, mais très exactement l'inverse. Partout, il a conduit à marginaliser et à exclure des populations entières qui avaient un rôle à jouer » (Maalouf, 2019, p. 80). Morin dira qu'« il y a sept savoirs "fondamentaux" que l'éducation du futur devrait traiter dans toute société comme dans toute culture, sans exclusive ni rejet, selon les modes et règles propres à chaque société et chaque culture» (Morin, 1999, p. 1). Ainsi compris, nous avons interprété certaines valeurs canadiennes et les discriminations systémiques selon un système de représentation construit autour de l'importance de promouvoir et d'enseigner l'éducation inclusive. Une valeur qui nous est chère pour l'éducation d'aujourd'hui et pour les générations à venir.

\section{BIBLIOGRAPHIE}

Bélanger, N. (2019). Diversité et minorités au prisme de réformes éducatives. Approche comparative de la 'révolution du bon sens' en Ontario (Canada) et des 'écoles de demain' en Nouvelle-Zélande. Éducation et sociétés. 44, 113-129.

Bergeron, C. (2019). L'importance de préserver la diversité des accents pour contrer l'insécurité linguistique en Ontario français. Alternative Francophone, 2(4), 92-107.

Bergeron, C., Blanchet, P. \& Lebon-Eyquem, M. (2020). La discrimination est présente partout, révèle une étude faite auprès d'étudiants canadiens et français. The Conversation Canada. https:// theconversation.com/la-discrimination-est-presente-partout-revele-une-etude-faite-aupresdetudiants-canadiens-et-francais-140497

Bissoondath, N. (1994). Selling Illusions: The Cult of Multiculturalism in Canada. Toronto : Penguin Books.

Blaya, C. (2011). Cyberviolence et cyberharcèlement : approches sociologiques. La nouvelle revue de l'adaptation et de la scolarisation, 1, 53, 47-65.

Bouchard, C. (2012). L’interculturalisme. Un point de vue québécois. Montréal : Les Éditions du Boréal. Bock-Côté, M. (2016). Le multiculturalisme comme religion politique. Paris : Les Éditions du Cerf.

Boltanski, L. (1990). Sociologie critique et sociologie de la critique. Politix. Revue des sciences sociales du politique, 10-11, 124-134. 
Colombain, J. (2019). Faut-il quitter les réseaux sociaux? Les cinq fléaux qui rongent Facebook, Twitter, Instagram, Snapchat et YouTube. Paris : Éditions DUNOD.

Commission ontarienne des droits de la personne. (2017). Rapport. Prendre le pouls. Opinion des gens à l'égard des droits de la personne en Ontario. Toronto : Gouvernement de l'Ontario.

Feireisen, C. (2020). Des Ontariens dénoncent une discrimination systémique au sein des institutions publiques. Radio-Canada. https://ici.radio-canada.ca/nouvelle/1711532/racismesystemique-fonction-publique-ontario

Garneau, S. (2010). Penser le pluralisme des francophonies minoritaires canadiennes : de la logique identitaire à la question sociale. Reflets, 16, 2, 22-56.

Gignac, J.-L. (1997). Sur le multiculturalisme et la politique de la différence identitaire : Taylor, Walzer, Kymlicka. Politique et Sociétés, 16, 2, 31-65.

Harris, S. (2020). Black Students Still Face ‘Toxic' Racism Despite Decades of 'Solutions' In Ontario. HuffPost. https://www.huffingtonpost.ca/entry/ontario-schools$\underline{\text { racism_ca_5efocad1c5b685770a16b072 }}$

Hubert, T. (2014). Un collégien sur cinq concerné par la « cyber-violence ». Note d'information no 39. https://www.education.gouv.fr/un-collegien-sur-cinq-concerne-par-la-cyberviolence-3815

Lepage, P. (2019). Mythes et réalités sur les peuples autochtones. Sept-îles, Québec : Institut Tshakapesh, $3^{\mathrm{e}}$ édition. http://www.cdpdj.qc.ca/Publications/Mythes-Realites.pdf? fbclid=IwAR00p36eQdB8pWCW_kB5Xtt9fYF9wvzjy210omkrb40b63fx4Sw1NIncFH8

LeVasseur, G. (2016). Discrimination linguistique. Le Droit. https://www.ledroit.com/opinions/ votre-opinion/discrimination-linguistique-e82271d168c53cd99cfc88233b51ac3e

Lewis, S. (1992). Report on Race Relations in Ontario. https://collections.ola.org/mon/ 13000/134250.pdf

Maalouf, A. (2019). Le naufrage des civilisations. Paris : Éditions Grasset \& Fasquelle.

Maalouf, A. (1998). Les identités meurtrières. Paris : Éditions Grasset \& Fasquelle.

MacLennan, H. (1945). Two Solitudes. Toronto : Macmillan of Canada.

Ministère de l'Éducation de l'Ontario. (2017). Plan d'action ontarien pour l'équité en matière d'éducation. http://www.edu.gov.on.ca/fre/about/education_equity_plan_fr.pdf

Ministère de l'Éducation de l'Ontario. (2014). Équité et éducation inclusive dans les écoles de l'Ontario. Lignes directrices pour l'élaboration et la mise en œuvre de politiques. http://www.edu.gov.on.ca/fre/ policyfunding/inclusiveguide.pdf

Ministère de l'Éducation de l'Ontario. (2009). Comment tirer parti de la diversité. Stratégie ontarienne d'équité et d'éducation inclusive. http://www.edu.gov.on.ca/fre/policyfunding/equity.pdf

Morin, E. (2005). Introduction à la pensée complexe, Paris : Éditions du Seuil, $2^{\mathrm{e}}$ édition.

Morin E. (1999). Les sept savoirs nécessaires à l'éducation du futur. Paris : Éditions du Seuil.

Morin, E. (1994). La complexité humaine. Textes rassemblés par Edgar Morin et présentés par Heinz Weinmann, Paris : Éditions Flammarion.

Morin, E. \& Le Moigne, J.-L. (1999). L'intelligence de la complexité, Paris : L'Harmattan.

Nations Unies. (2013). Deuxième Commission: la mondialisation et l'interdépendance qu'elle crée entre nations doivent être soutenues par un partenariat de développement plus équitable. https:// www.un.org/press/fr/2013/AGEF3375.doc.htm 
Orwell, G. (1984). La ferme des animaux. Paris : Éditions Gallimard.

Paugam, S. (2008). La pratique de la sociologie. Paris : Presses universitaires de France.

Radio-Canada (2020). Des Noirs francophones souhaiteraient plus de reconnaissance des autres Franco-Ontariens. Radio-Canada. https://ici.radio-canada.ca/nouvelle/1710957/noirs-ontariofrancais-reocnnaissance-black-lives-matter-racisme

Radio-Canada (2019). Les élèves canadiens parmi les meilleurs du monde en mathématiques et en sciences. Radio-Canada. https://ici.radio-canada.ca/nouvelle/1414836/performance-elevescanada-test-pisa-ocde

Ratel, M.-H. (2020). Le manque de diversité à l'AEFO critiqué. Radio-Canada. https://ici.radiocanada.ca/nouvelle/1716279/aefo-franco-ontariens-inclusion-diversite-critiques

Robert, P. (2009). Une théorie sociétale des TIC. Penser les TIC entre approche critique et modélisation conceptuelle. Paris : Éditions Lavoisier.

Saez, J.-P. (2008). Vitalité de la diversité culturelle et mondialisation. Entretien avec Edgar Morin. Observatoire des politiques culturelles, 1(33), 3-8.

Sauvageau, F. Thibault, S. \& Trudel, P. (2018). Les fausses nouvelles, nouveaux visages, nouveaux défis. Comment déterminer la valeur de l'information dans les sociétés démocratiques? Québec : Les Presses de l'Université Laval.

Slaughter, G. \& Singh, M. (2020). Five charts that show what systemic racism looks like in Canada. CTV News. https://www.ctvnews.ca/canada/five-charts-that-show-what-systemic-racism-lookslike-in-canada-1.4970352

Statistique Canada (2017a). Immigration et diversité ethnoculturelle : faits saillants du Recensement de 2016 https://www150.statcan.gc.ca/n1/daily-quotidien/171025/dq171025b-fra.pdf

Statistique Canada (2017b). Les origines ethniques et culturelles des Canadiens, le portrait d'un riche héritage. Recensement de 2016 https://www12.statcan.gc.ca/census-recensement/2016/as-sa/ 98-200-x/2016016/98-200-x2016016-fra.cfm

Taylor, C. (2009). Multiculturalisme : différence et démocratie. Montréal : Éditions Flammarion.

UNESCO. (2001). Déclaration universelle de l'UNESCO sur la diversité culturelle. http:// portal.unesco.org/fr/ev.php-URL_ID=13179\&URL_DO=DO_TOPIC\&URL_SECTION=201.html

Vachet, B. (2020). L'insécurité linguistique, conséquence d'une discrimination ? ONFR+. https:// onfr.tfo.org/linsecurite-linguistique-consequence-dune-discrimination/

Yvon, A.-M. (2020). Haro sur le racisme envers les Autochtones. Radio-Canada. https://ici.radiocanada.ca/espaces-autochtones/1499861/racisme-autochtones-maitee-labrecque-saganashwidia-lariviere-isabelle-picard

\section{NOTES}

1. Avec l'autorisation de l'étudiant (immigrant francophone), nous nous permettons de partager son expérience dans le cadre de sa formation en enseignement : «Au début des cours, je m'étais attendue à un cours sur l'histoire franco-ontarienne avec un aperçu de la démographie des élèves dans les écoles francophones en Ontario. Cependant, ce fut une expérience de rétrospection. À travers les lectures et les discussions en classe, je me suis aperçue qu'il y avait une place pour moi dans cette communauté minoritaire francophone en Ontario. De plus, j'ai compris davantage la complexité des enjeux qui la composent, et de comment l'appartenance à cette communauté 
signifie aussi une responsabilité. Mon attitude par rapport à la communauté francophone a changé, lâchant cette sensation d'être un imposteur pour me laisser accueillir au sein du groupe.»

2. Pour plus de détails sur les deux modèles, le lecteur est invité à consulter les ouvrages du sociologue Gérard Bouchard (2012) et du philosophe Charles Taylor (2009). Pour une critique du multiculturalisme, le lecteur peut consulter les ouvrages du sociologue Mathieu Bock-Côté (2016) et de l'essayiste Neil Bissoondath (1994).

3. « Tous les animaux sont égaux, mais certains le sont plus que d'autres. »

\section{RÉSUMÉS}

La démographie canadienne est plurielle et contrastée. L'un des défis de la société est de tirer profit de cette diversité, mais de nombreuses discriminations existent. Depuis quelques années, l'éducation inclusive est valorisée dans les écoles ontariennes. Dans cet article, nous interprétons l'éducation inclusive à l'aide de la pensée complexe d'Edgar Morin sous le prisme de l'enseignement de l'identité terrienne et de la compréhension. Sans être une recette miracle, l'éducation inclusive peut jouer un rôle actif dans l'élimination des discriminations.

The Canadian demographic is plural and contrasted. One of society's challenges is to take advantage of this diversity, but many discriminations exist. These last couple years, inclusive education is highly valued in Ontarian schools. In this article, we will interpret inclusive education with the help of the complex thought from Edgar Morin under the prism of the education of "terrienne" identity and comprehension. Without being a miracle solution, inclusive education can play an active role in the elimination of discriminations.

\section{INDEX}

Mots-clés : éducation inclusive, Edgar Morin, théorie de la complexité, discriminations

Keywords : inclusive education, Edgar Morin, theory of complexity, discriminations

\section{AUTEUR}

\section{CHRISTIAN BERGERON}

Faculté d'éducation, Université d'Ottawa, Canada 